\title{
Internal static electric and magnetic field at the copper cite in a single crystal of the electron-doped high- $\mathrm{T}_{c}$ superconductor $\operatorname{Pr}_{1.85} \mathrm{Ce}_{0.15} \mathrm{CuO}_{4-y}$
}

\author{
Guoqing Wu, ${ }^{1,2}$ F. Zamborszky, ${ }^{2}$ A. P. Reyes,${ }^{3}$ P. L. Kuhns,${ }^{3}$ R. L. Greene,${ }^{4}$ and W. G. Clark ${ }^{2}$ \\ ${ }^{1}$ College of Physics Science and Technology, Yangzhou University, Yangzhou, Jiangsu 225002, China \\ ${ }^{2}$ Department of Physics and Astronomy, UCLA, Los Angeles, California 90095, USA \\ ${ }^{3}$ National High Magnetic Field Laboratory, Florida State University, Tallahassee, Florida 32306, USA and \\ ${ }^{4}$ Department of Physics, University of Maryland, College Park, Maryland 20742, USA
}

(Dated: July 13, 2021)

\begin{abstract}
We report ${ }^{63,65} \mathrm{Cu}-\mathrm{NMR}$ spectroscopy and Knight shift measurements on a single crystal of the electron-doped high- $T_{c}$ superconductor $\mathrm{Pr}_{1.85} \mathrm{Ce}_{0.15} \mathrm{CuO}_{4-y}$ (PCCO) with an applied magnetic field $(H)$ up to 26.42 T. A very small NQR frequency is obtained with the observation of the spectrum, which shows an extremely wide continuous distribution of it that becomes significant narrower below $20 \mathrm{~K}$ at $H \| c$ where the superconductivity is completely suppressed, indicating a significant change in the charge distribution at the $\mathrm{Cu}$ site, while the corresponding changes at $H \perp c$ is negligible when the superconductivity is present or not fully suppressed. The Knight shift and central linewidth are proportional to the applied magnetic field with a high anisotropy. We find that the magnitude of the internal static magnetic field at the copper is dominated by the anisotropic $\mathrm{Cu}^{2+} 3 d$-orbital contributions, while its weak temperature-dependence is mainly determined by the isotropic contact hyperfine coupling to the paramagnetic $\operatorname{Pr}^{3+}$ spins, which also gives rise to the full distribution of the internal static magnetic field at the copper for $H \perp c$. This internal static electric and magnetic field environment at the copper is very different from that in the hole-doped cuprates, and may provide new insight into the understanding of high- $T_{c}$ superconductivity. Other experimental techniques are needed to verify whether the observed significant narrowing of the charge distribution at the $\mathrm{Cu}$ site with $H \| c$ is caused by the charge ordering (CO) [E. H. da Silva Neto et al., to be published in Science $]^{1}$ or a new type of charge modulation.
\end{abstract}

PACS numbers: 74.72.Jt., 74.25.Jb, 74.25.Nf, 76.60-k

\section{INTRODUCTION}

Understanding the mechanism of superconductivity has been an outstanding challenge in physics. The recently advanced technique of resonant inelastic Xray scattering experiments ${ }^{2}$ in hole-doped cuprate high$T_{c}$ (where $T_{c}$ is the temperature for the superconducting phase transition) superconductors (HTSCs) $\mathrm{La}_{2-x} \mathrm{Sr}_{x} \mathrm{CuO}_{4}$ (LSCO) does not support the paring by exchange of magnetic excitations ${ }^{3}$ as one of the most intensely studied scenarios of high- $T_{c}$ superconductivity, while the proposal $\underline{\underline{4}}^{\underline{4}}$ of spin fluctuations associated with a magnetic interaction between planar quasiparticles (in the $\mathrm{CuO}_{2}$-plane) in hole-doped cuprate HTSCs seems to be largely supported. For example, the latter is thought to be responsible for both the anomalous normal state behavior (which strongly deviates from the Fermi liquid theory $)^{\frac{5}{5}}$ and the transition to a superconducting state with an anisotropic orbital $d_{x^{2}-y^{2}}$ wave paring symmetry $\underline{\underline{6}, 7}$ However, none of them is conclusive $\underline{\underline{8}}$

Recent experimental evidence favors a competing scenario in the hole-doped cuprate HTSCs of LSCO,,-11 $\mathrm{YBa}_{2} \mathrm{Cu}_{3} \mathrm{O}_{y} \quad(\mathrm{YBCO})^{12-15}$ and $\mathrm{Bi}_{2} \mathrm{Sr}_{2} \mathrm{CaCu}_{2} \mathrm{O}_{6+y}$ (BSCCO) $\stackrel{16,17}{1}$ which show a competition between superconductivity and other long-range ordered phases, such as a charge-density wave (CDW) $, \underline{9}-11,13$ charge ordering (CO), 13,16,18 and/or even antiferromagnetic (AFM) order, $\frac{19}{9}$ etc., with the tuning of the applied magnetic field and/or hole-doping level. This interplay between competing phases is also partly observed in the electron- doped cuprate HTSCs $\mathrm{Nd}_{2-x} \mathrm{Ce}_{x} \mathrm{CuO}_{4-y}$ (NCCO), $\stackrel{20}{,}$ and in the Fe-based high- $T_{c}$ superconductors 21 as well as in the newly discovered Ti-based ${ }^{22.23}$ superconductors.

Unlike the hole-doped cuprate HTSCs, the electrondoped cuprate HTSCs $R_{2-x} \mathrm{M}_{x} \mathrm{CuO}_{4-y}$ (RMCO, $R=$ $\mathrm{Nd}, \mathrm{Pr}, \mathrm{Eu}, \mathrm{Sm}, \mathrm{La} ; \mathrm{M}=\mathrm{Ce}, \mathrm{Th})^{24.25}$ show a larger area of antiferromagnetism with no pseudogap phase in the underdoped regime of the phase diagram, 26 have a substantially lower value of $T_{c}$ (optimal $T_{c} \sim 25 \mathrm{~K}$ ), and have a smaller value of upper critical field $\left(H_{c 2}\right)^{27}$ than their hole-doped counterparts. These differences suggest 25.28 the significance of the antiferromagnetic spin fluctuations which are related to the internal magnetic field environment at the $\mathrm{Cu}$ site. On the other hand, in structure they have a slightly longer in-plane $\mathrm{Cu}-\mathrm{O}$ bond length that might be associated with their lack of apical oxygen for the $\mathrm{T}^{\prime}$-structure of their crystal lattice, in contrast to the T-structure of their hole-doped counterpart

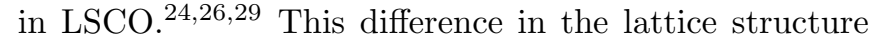
may also have a direct impact on the internal electric and magnetic field environment at the copper which could ultimately determine their spin fluctuations and electron paring. $3.4,8,25,29$ Therefore, it is important to study the internal electric and magnetic field at the copper in the $\mathrm{CuO}_{2}$-plane in these materials. Moreover, this local field environment reveals their intrinsic properties, including the sources of the charge and spin dynamics ${ }^{30}$ of the $\mathrm{Cu}-$ $3 \mathrm{~d}$ conduction electrons.

NMR (nuclear magnetic resonance) has played a key role in these local field determinations, with intensive 
studies carried on the hole-doped cuprate HTSCs $31-34$ For example, recent NMR studies of the internal static electric and magnetic field on $\mathrm{LSCO}^{35}$ and $\mathrm{YBCO}^{36}$ have found $\mathrm{CO}$, where the hole-doped charges are modulating around the vortex cores, providing evidence of $\mathrm{CO}$ that competes with the superconductivity and suggesting a possible relationship to the electron paring.

But there are very few NMR reports on the electrondoped cuprate HTSCs, which were mainly for measurements on powder samples. For example, NMR measurements on powder samples 37 of $\mathrm{Pr}_{2-x} \mathrm{Ce}_{x} \mathrm{CuO}_{4-y}$ (PCCO, $x=0.10,0.15$ and 0.20 ) by Williams et al. were focusing on spin dynamic properties and show conflicting aspects, such as no doping dependence of the ${ }^{63} \mathrm{Cu}-\mathrm{NMR}$ spinlattice relaxation as a reflection of the internal magnetic field fluctuations at the copper, which are against the widely accepted theoretical predictions ${ }^{38}$ Thus the intrinsic properties of the electron-doped cuprate HTSCs remain elusive. Since single crystals have lots of advantages over powder samples, it is necessary to examine these properties using single crystals. Recent NMR measurements 39,40 on single crystals of PCCO show the effect of doping to the number of hole contents in the $\mathrm{Cu}$ $3 \mathrm{~d}$ and $\mathrm{O} 2 \mathrm{p}$ orbitals and to the ${ }^{63} \mathrm{Cu}-\mathrm{NMR}$ quadrupole splitting frequency. However the important information regarding the distribution of the spectrum satellites and their temperature dependence is still missing.

In this paper, we report ${ }^{63,65} \mathrm{Cu}$-NMR spectroscopy and Knight shift measurements on a single crystal of the electron-doped cuprate HTSC PCCO with an applied magnetic field $(H)$ up to $26.42 \mathrm{~T}$, at which the superconductivity at $H \| c$ is completely suppressed,, 27 so that the normal state static local field properties at the copper can be evaluated down to low temperatures.

Our main results are that a very small NQR frequency $\nu_{Q} \sim 2.2 \mathrm{MHz}$ is obtained with the observation of an unusual ${ }^{63,65} \mathrm{Cu}-\mathrm{NMR}$ spectrum, which shows a very small electric field gradient (EFG) (corresponding to the value of $\nu_{Q}$ ) and an extremely wide continuous distribution of it $\left(\Delta \nu_{Q} \sim 18 \mathrm{MHz}\right)$ at the copper site in PCCO. The distribution becomes significantly narrower below $20 \mathrm{~K}$ at $H \|$ $c$ where the superconductivity is completely suppressed, indicating a significant change in the charge distribution at the $\mathrm{Cu}$ site which may be associated with the $\mathrm{CO}$ most recently found in the optimally doped NCCO $\frac{1}{-}$ while the corresponding changes at $H \perp c$ are negligible when the superconductivity is present or not fully suppressed. The ${ }^{63,65} \mathrm{Cu}-\mathrm{NMR}$ Knight shift and the central linewidth are proportional to $H$ with a high anisotropy. We find that the magnitude of the internal static magnetic field at the copper is dominated by the anisotropic $\mathrm{Cu}^{2+} 3 d$-orbital contributions, but its weak temperature $(T)$ dependence is mainly determined by the isotropic contact hyperfine coupling to the paramagnetic $\mathrm{Pr}^{3+}$ electron spins, which also generates essentially the full distribution of the internal static magnetic field at the copper at $H \perp c$. This internal static electric and magnetic field environment at the copper in the electron-doped cuprate HTSCs is very different from that in their hole-doped counterparts, where there is no evidence of a contribution from ions with a large spin paramagnetic moment.

\section{EXPERIMENTAL DETAILS}

The high quality single crystal of PCCO (optimaldoped) was grown with a flux technique $\frac{41,42}{}$ and annealed in argon at $900{ }^{\circ} \mathrm{C}$ for $48 \mathrm{~h}$. The sample size is $1.5 \mathrm{~mm} \times 1.2 \mathrm{~mm} \times 35 \mu \mathrm{m}$ with a mass of $0.53 \mathrm{mg}$. The NMR coil was made from $50 \mu \mathrm{m}$ diameter silver wire wound with $\sim 20$ turns, and fixed with epoxy in order to get rid of "coil disease" (phonon assisted vibrations). The coil, with the sample in it, was mounted on a small platform that is attached to a goniometer that is rotated about a sample axis. The sample is oriented with the rotation axis in the $a b$-plane and perpendicular to the applied magnetic field $H$ so that the angle $(\Theta)$ between the lattice $c$-axis and $H$ can vary as the sample rotates (note, the crystal lattice of PCCO is tetragonal).

The ${ }^{63,65} \mathrm{Cu}-\mathrm{NMR}$ frequency-swept and field-swept spectra were obtained using standard spin-echo techniques carried out with a spectrometer and probe built at UCLA (W. G. Clark) for field $H=9 \mathrm{~T}$ and at the National High Magnetic Field Laboratory (NHMFL) in Florida for field $H=26.42 \mathrm{~T}$, respectively. Since the gyromagnetic ratio $\gamma_{I}$ for the ${ }^{63} \mathrm{Cu}$ is ${ }^{63} \gamma_{I}=11.285 \mathrm{MHz} / \mathrm{T}$ and for the ${ }^{65} \mathrm{Cu}$ is ${ }^{65} \gamma_{I}=12.089 \mathrm{MHz} / \mathrm{T}$, the frequency $\nu$ for the excitation pulses used for the spectrometer is near $\nu \sim \nu_{0}=\gamma_{I} H=\sim 102(\mathrm{MHz})$ and $\sim 298(\mathrm{MHz})$ for the ${ }^{63} \mathrm{Cu}$ at $H=9 \mathrm{~T}$ and $H=26.42 \mathrm{~T}$, respectively, where $\nu_{0}$ is the ${ }^{63} \mathrm{Cu}$ Larmor frequency in the external field. The corresponding values for the ${ }^{65} \mathrm{Cu}$ are $\sim 109$ $(\mathrm{MHz})$ and $\sim 319(\mathrm{MHz})$ at $H=9 \mathrm{~T}$ and $H=26.42 \mathrm{~T}$, respectively.

Since the ${ }^{63,65} \mathrm{Cu}-\mathrm{NMR}$ spectrum covers a very wide range in frequency up to $\sim 18 \mathrm{MHz}(1.5 \mathrm{kG})$ at all temperatures (and fields), short rf pulses and a wide receiver bandwidth $( \pm 1 \mathrm{MHz})$ were used to record the spin-echo signals. The pulse sequence that optimized the height of the ${ }^{63} \mathrm{Cu}-\mathrm{NMR}$ spin echo (with the central line) used for most of the NMR signal recording was a $0.6 \mu \mathrm{s} \pi / 2$ pulse $\left(p_{1}\right)$ [ i.e., rf field $H_{1}=1 /\left(4^{63} \gamma p_{1}\right)=369 \mathrm{G}$, or $0.42 \mathrm{MHz}{ }^{63} \mathrm{Cu}$ frequency ] followed by a $1.0 \mu \mathrm{s} \pi$-pulse $\left(p_{2}\right)$ separated by a time interval $\tau(\tau \sim 10 \mu \mathrm{s})$ for most of the measurements at both $H=9 \mathrm{~T}$ and $H=26.42$ $\mathrm{T}$ using the same NMR sample coil (note, the optimized pulses for the ${ }^{65} \mathrm{Cu}$ spin echo is rather similar). For a viable signal-to-noise ratio, each echo signal was averaged 1000 times at $200 \mathrm{~K}$ and 64 times at $10 \mathrm{~K}$ and lower temperatures at $H=9 \mathrm{~T}$, while at $H=26.42 \mathrm{~T}$ the corresponding number of averages used in the measurements is $\sim 4$ times smaller.

At $H=9 \mathrm{~T}$, the typical range of the frequency sweep covered $20 \mathrm{MHz}$ (from $95 \mathrm{MHz}$ to $115 \mathrm{MHz}$ ) at all temperatures, and it used a frequency step $0.1 \mathrm{MHz}$ for each spin-echo acquisition. In order to maintain a uniform 
high sensitivity (above 85\%), the probe circuit was first tuned to $95.5 \mathrm{MHz}$, and then retuned manually every $1 \mathrm{MHz}$ (i.e., 10 acquisitions) for the spectrum recording. The frequency-swept spectra were analyzed with the frequency-shifted and -summed (FrSS) Fourier transform processing $\underline{\underline{43}}$

At $H=26.42 \mathrm{~T}$, the range of the field sweep for the ${ }^{63} \mathrm{Cu}$-NMR spin-echo signal covered $0.86 \mathrm{~T}$ (from 26.0 $\mathrm{T}$ to $26.86 \mathrm{~T})$ at all temperatures with a fixed NMR frequency $\left(\nu_{0}=298.16 \mathrm{MHz}\right)$ from the frequency generator, and the sweep used a field step $0.02 \mathrm{~T}$ ( i.e., $0.226 \mathrm{MHz}$ in frequency ) for each spin-echo acquisition. The recording for the ${ }^{65} \mathrm{Cu}$-NMR spin-echo signals was similar. The field-swept spectra were analyzed with the field-shifted and -summed (FiSS) Fourier transform processing. $\underline{\underline{43}}$

The corresponding ${ }^{63,65} \mathrm{Cu}-\mathrm{NMR}$ Knight shift and central linewidth at $H=9 \mathrm{~T}$ and $H=26.42 \mathrm{~T}$ were obtained from the frequency- and field-swept spectra as described above.

For the purposes of the applied field calibrations, a small piece of thin Al-foil was inserted into the sample coil with the PCCO sample [ note, the ${ }^{27} \mathrm{Al}$ nucleus in the Al-foil has a gyromagnetic ratio ${ }^{27} \gamma=11.0943 \mathrm{MHz} / \mathrm{T}$ and an isotropic Knight shift ${ }^{27} K=0.162 \%$, i.e., the ${ }^{27} \mathrm{Al}$ has an effective gyromagnetic ratio ${ }^{27} \gamma_{\text {eff }}=(1+$ $0.162 \%) \times 11.0943=11.112 \mathrm{MHz} / \mathrm{T}]$.

Also we did the DC magnetic susceptibility measurements with an applied magnetic field 3000 Oe upon cooling in temperature from $300 \mathrm{~K}$ down to $2 \mathrm{~K}$, using a commercial SQUID magnetometer.

\section{RESULTS}

\section{A. ${ }^{63,65} \mathbf{C u}-\mathrm{NMR}$ spectra}

Figure 1 shows the ${ }^{63,65} \mathrm{Cu}-\mathrm{NMR}$ spectra with an applied magnetic field $H=9 \mathrm{~T}$ at a typical temperature $T=50 \mathrm{~K}$, plotted as the ${ }^{63,65} \mathrm{Cu}-\mathrm{NMR}$ spin-echo amplitude vs frequency shift $\nu-\nu_{r f}$, where $\nu_{\mathrm{rf}}$ is a reference frequency (here $\nu_{\mathrm{rf}}=106 \mathrm{MHz}$ ). The area of each spectrum curve (above its baseline) at both $H \| c$ and $H \perp c$ is normalized to be 1 for comparison. Theoretically each copper nucleus's spectrum is expected to have a central line plus two symmetric quadrupolar satellites due to the ${ }^{63,65} \mathrm{Cu}$ spin quantum $m=+1 / 2 \leftrightarrow-1 / 2$ (central) and $\pm 3 / 2 \leftrightarrow \pm 1 / 2$ (satellites) transitions, respectively.

Instead of sharp satellite spectra, the satellite spectra are extremely broad, with structures that spread across the sharp central lines and overlap between them. The overlap also extends significantly between the two copper isotopes, especially at $H \| c$, and their spectra totally cover a range of $\sim 18 \mathrm{MHz}$ in frequency. Interestingly, the quadrupolar satellites become narrower and the their peaks become observable (see Fig. 2) upon cooling in temperatures below $\sim 20 \mathrm{~K}$. The ratio for the areas below each spectrum curve for each $\mathrm{Cu}$ isotope for the satellites and central line in total is $\sim 55 / 45$, which is close to the

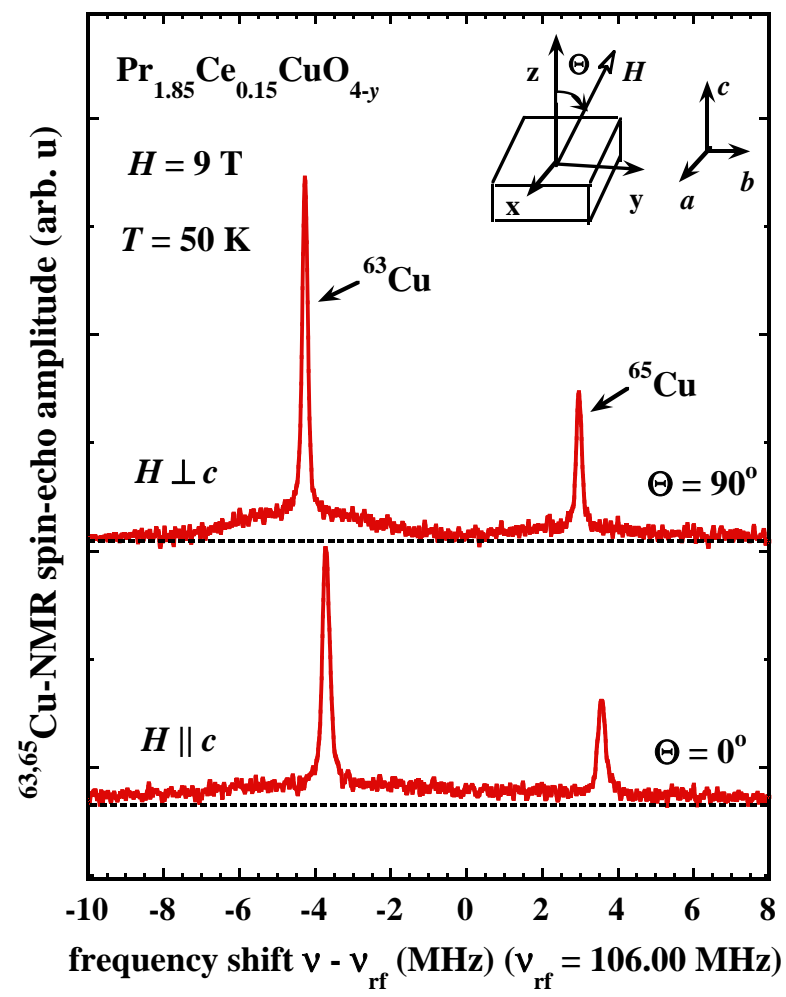

FIG. 1: (Color online) ${ }^{63,65} \mathrm{Cu}-\mathrm{NMR}$ frequency-swept spectra of a single crystal $\operatorname{Pr}_{1.85} \mathrm{Ce}_{0.15} \mathrm{CuO}_{4-y}(\mathrm{PCCO})$ at $T=50$ $\mathrm{K}$ with $H=9 \mathrm{~T}$. The dashed lines are the baselines for the spectra. The upper right corner indicates the lattice axis directions and the direction of $H$ in the $y z$-plane with an angle $\Theta$ relative to the $c$-axis of the thin plate-like crystal sample. The value of $\nu_{\mathrm{rf}}$ is a reference frequency used for the plot.

theoretically expected value $60 / 40, \underline{30}$

Theoretically, in the high field limit where the Zeeman splitting (Hamiltonian $H_{\text {Zeeman }}=-\gamma_{I} \hbar \overrightarrow{\mathbf{I}} \cdot \overrightarrow{\mathbf{H}}$ ) is dominant, for a spin $I=3 / 2$ nucleus the central line has a quadrupolar frequency shift to the 2 nd order as ${ }^{30} \Delta \nu_{c Q}^{(1)}$ $=0$, and

$$
\Delta \nu_{c Q}^{(2)}=\frac{3 \nu_{Q}^{2}}{16 \nu_{0}}\left(1-\cos ^{2} \Theta\right)\left(1-9 \cos ^{2} \Theta\right),
$$

while the two satellites have the 1st order quadrupolar frequency shifts 30

$$
\Delta \nu_{s Q}^{(1)}=\frac{\nu_{Q}}{2}\left(3 \cos ^{2} \Theta-1\right),
$$

arising from the electric quadrupole interaction of the nuclear quadrupolar moment $(Q)$ with the EFG, where $\nu_{Q}=e Q V_{z z} / 2 h$, called the nuclear quadrupolar resonance (NQR) frequency, $h$ is the Planck constant, $e$ is the charge of an electron, and $\nu_{0}=\gamma_{I} H$. The value of $Q$ for ${ }^{63} \mathrm{Cu}$ is ${ }^{63} Q=-0.211$ barns, and for ${ }^{65} \mathrm{Cu}$ it is ${ }^{65} Q=-0.195$ barns (note, 1 barn $=10^{-28} \mathrm{~m}^{2}$ ). Here the principle axes of the EFG $\left(V_{x x}, V_{y y}\right.$, and $\left.V_{z z}\right)$ at the $\mathrm{Cu}$ site can be chosen along the lattice $a, b$ and $c$ axes, 


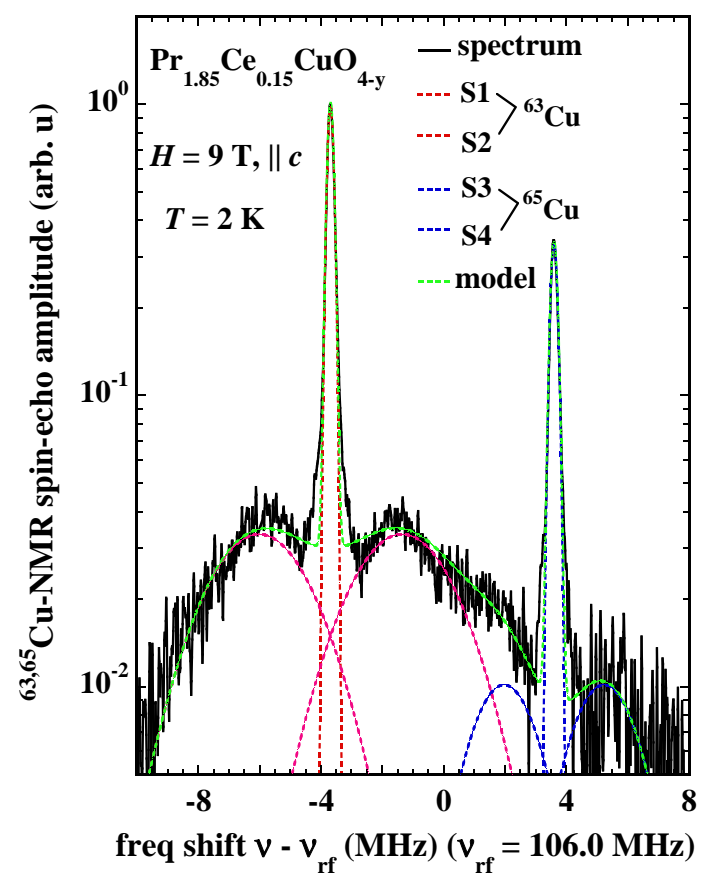

FIG. 2: (Color online) The fit of the full ${ }^{63,65} \mathrm{Cu}-$ NMR frequency-swept spectra of a single crystal $\operatorname{Pr}_{1.85} \mathrm{Ce}_{0.15} \mathrm{CuO}_{4-y}$ (PCCO) at temperature $T=2 \mathrm{~K}$ with $H=9 \mathrm{~T}, \| c$, using a Gaussian model. The dashed red and blue curves are the fit for the satellites of ${ }^{63} \mathrm{Cu}$ and ${ }^{65} \mathrm{Cu}$, respectively, and the dashed green curves are the fit total from the model as compared with the measured spectrum (the solid black curve) (for convenience, the vertical axis is plotted using a logarithmic scale).

respectively, and then the anisotropic EFG tensor $\eta=$ $\left(V_{x x}-V_{y y}\right) / V_{z z}=0$, due to the tetragonal lattice symmetry.

With the analysis using Eqs. (1)-(2) for the angular dependence of the ${ }^{63,65} \mathrm{Cu}-\mathrm{NMR}$ spectra, we found ${ }^{63} \nu_{Q}$ $=2.17 \pm 0.03 \mathrm{MHz}$ for the ${ }^{63} \mathrm{Cu}$, and ${ }^{65} \nu_{Q}=2.08 \pm 0.04$ $\mathrm{MHz}$ for the ${ }^{65} \mathrm{Cu}$. This gives an experimental ratio of ${ }^{63} \nu_{Q} /{ }^{65} \nu_{Q} \approx 1.05$, which also agrees with the theoretically equivalent ratio of ${ }^{63} Q /{ }^{65} Q=1.08 \cdot{ }^{30}$

However, this value of $\nu_{Q}$ for the ${ }^{63,65} \mathrm{Cu}$ in PCCO is very small, and the ${ }^{63,65} \mathrm{Cu}-\mathrm{NMR}$ spectrum satellites are extremely broad, in comparison with those in the hole-doped counterparts LSCO $\left({ }^{63} \nu_{Q} \sim 35 \mathrm{MHz}\right) \stackrel{31}{\underline{3}}$ and YBCO $\left({ }^{63} \nu_{Q}=31 \mathrm{MHz}\right), 32$ or its parent compound $\mathrm{Pr}_{2} \mathrm{CuO}_{4-y}(\mathrm{PCO})\left({ }^{63} \nu_{Q} \sim 15 \mathrm{MHz}\right) \stackrel{44}{\rightleftharpoons}$ which is an antiferromagnet. Similar observations were also obtained in the electron-doped compound $\mathrm{NCCO}^{45}$ which has a value of ${ }^{63} \nu_{Q} \sim 1 \mathrm{MHz}$ according to our estimate, while its parent compound $\mathrm{Nd}_{2} \mathrm{CuO}_{4-y}$ (NCO) (also an antiferromagnet) has a value of ${ }^{63} \nu_{Q} \sim 14 \mathrm{MHz}, \underline{45}, 46$ Thus the case for PCCO here is rather similar to that in NCCO.

It is not clear here what is the cause of the very small value of $\nu_{Q}$ (or EFG) and the extremely wide distribution of it at the $\mathrm{Cu}$ in PCCO and $\mathrm{NCCO} \stackrel{45,46}{=}$ whether they are doping or structure related, for example, even though

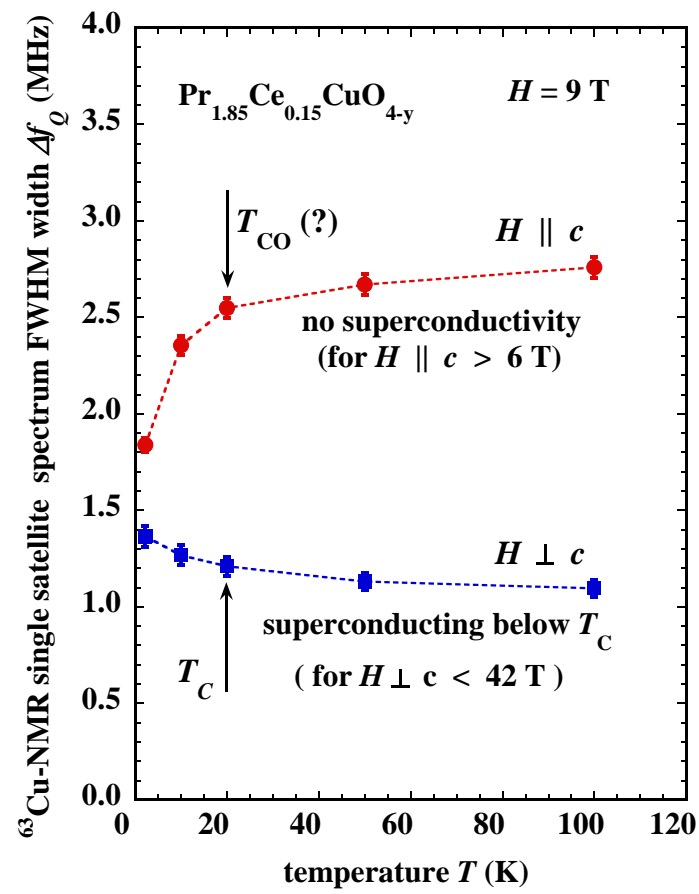

FIG. 3: (Color online) $\mathrm{T}$-dependence of the ${ }^{63} \mathrm{Cu}-\mathrm{NMR}$ single satellite spectrum FWHM width of $\operatorname{Pr}_{1.85} \mathrm{Ce}_{0.15} \mathrm{CuO}_{4-y}$ (PCCO) at $H=9 \mathrm{~T}$. The downward arrow indicates the temperature where a significant drop of the satellite linewidth starts (labeled as $T_{C O}$ for possible charge ordering) for $H \| c$ when the superconductivity is fully suppressed.

a similar value for $\nu_{Q}$ was theoretically estimated $\frac{47}{}$ by considering the covalence of $\mathrm{Cu}$ and overlapping of the electronic orbitals of $\mathrm{Cu}$ and $\mathrm{O}$, as well as the amount of $\mathrm{Cu}^{1+}$ impurity associated with the electron doping.

Figure 2 shows that the full ${ }^{63,65} \mathrm{Cu}-\mathrm{NMR}$ frequencyswept spectra of PCCO can be fitted using a Gaussian model, with high symmetry satellites for each isotope, with the one at temperature $T=2 \mathrm{~K}$ and $H=9 \mathrm{~T}, \| c$, as an example. The fit may not be physical, but it does provide a convenient way of obtaining the satellite width and the peak positions.

Figure 3 exhibits the $T$-dependence of the PCCO ${ }^{63} \mathrm{Cu}-\mathrm{NMR}$ single satellite spectrum full-width-halfmaximum (FWHM) width at an applied magnetic field $H=9 \mathrm{~T}$. These data indicate a significant narrowing of the satellites at $H \| c$, which may indicate a significant charge distribution modification at the $\mathrm{Cu}$ site at $T \leq$ $T_{C O}=20 \mathrm{~K}$ where the superconductivity is fully suppressed (with $H \| c \geq H_{c 2}=6 \mathrm{~T}$ ), while the corresponding change at $H \perp c$ is negligible when the superconductivity is present or not fully suppressed. This observation may serve as the evidence of the $\mathrm{CO}$ as recently found in the electron-doped cuprate $\mathrm{NCCO}, \frac{1}{,}$ while other experimental techniques are still needed for a verification.

Figure 4 shows the ${ }^{63} \mathrm{Cu}-\mathrm{NMR}$ spectrum at $H=26.42$ $\mathrm{T}$ as compared with that at $H=9 \mathrm{~T}$ at temperature $T=$ $4 \mathrm{~K}$. The spectra are normalized in area and plotted on top of each other by choosing different values of reference 


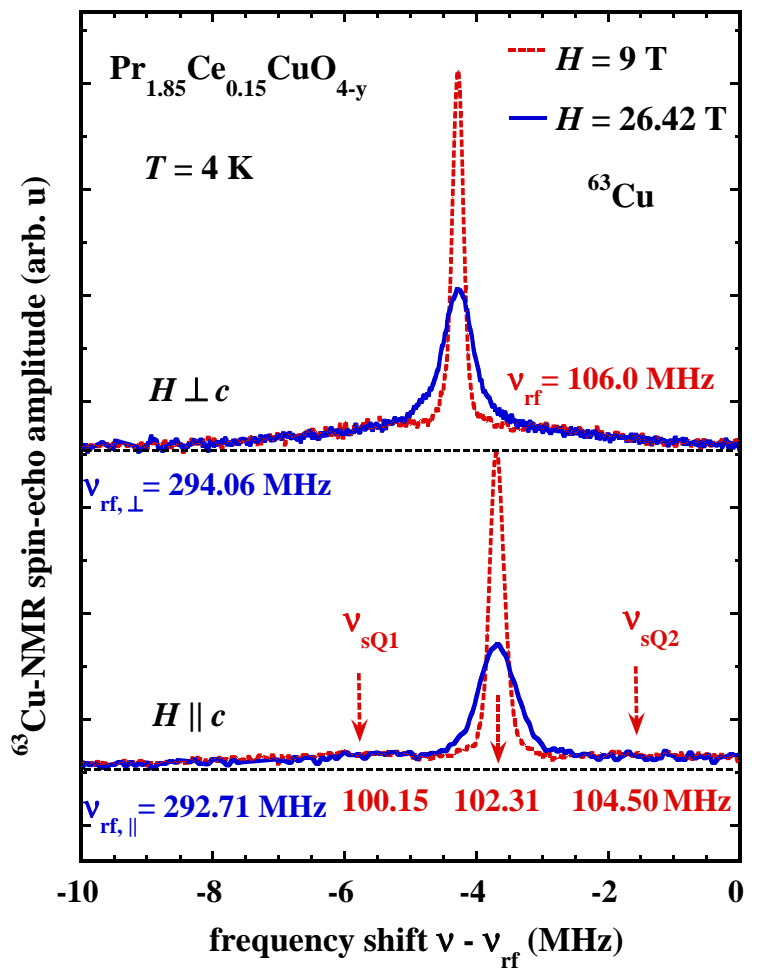

FIG. 4: (Color online) ${ }^{63} \mathrm{Cu}-\mathrm{NMR}$ frequency-swept spectra at $H=9 \mathrm{~T}$ (dashed red curves) and field-swept spectra at $H=26.42 \mathrm{~T}$ (solid blue curves) of a single crystal $\operatorname{Pr}_{1.85} \mathrm{Ce}_{0.15} \mathrm{CuO}_{4-y}(\mathrm{PCCO})$ at $T=4 \mathrm{~K}$. Different values of the reference frequency $\nu_{\mathrm{rf}}$ are used for the plot. The dashed (red) arrows indicate the satellite peak positions at $H \| c$.

frequency $\nu_{\mathrm{rf}}$ (note, here $\nu_{\mathrm{rf}}=106 \mathrm{MHz}$ for both $H \perp c$ and $H \| c$ at $9 \mathrm{~T}$ ). This indicates that the satellites at $H$ $=26.42 \mathrm{~T}$ are essentially the same as those at $H=9 \mathrm{~T}$, i.e., no $H$-dependence, and the full width half maximum (FWHM) central linewidth is $\sim 3$ times wider at $H=$ $26.42 \mathrm{~T}$ than at $H=9 \mathrm{~T}$, i.e., $\sim$ proportional to the applied magnetic field $H$.

Therefore, this reveals the origin of the internal static electric and magnetic field at the $\mathrm{Cu}$ : the satellites are due to the quadrupolar contribution with the charges surrounding the $\mathrm{Cu}$ site, while the central transition line is magnetic.

\section{B. ${ }^{63} \mathrm{Cu}-\mathrm{NMR}$ Knight shift}

Figure 5 shows the $T$-dependence of the ${ }^{63} \mathrm{Cu}-\mathrm{NMR}$ Knight shift, ${ }^{63} K(T)$ vs $T .{ }^{63} K(T)$ is highly anisotropic and has a fairly weak $T$-dependence at both $H \perp c$ and $H \| c$, and there is no significant change of ${ }^{63} K(T)$ across $T_{c}$ (at $H \perp c$ ) upon cooling. The superconductivity at $H \| c$ is completely suppressed by the applied magnetic field $H$ (when $H \geq 6 \mathrm{~T}$ ) $\stackrel{27}{=}$ Moreover, the proportionality of ${ }^{63} K(T)$ in frequency with $H$ further confirms the magnetic origin for the shift of the central line. The

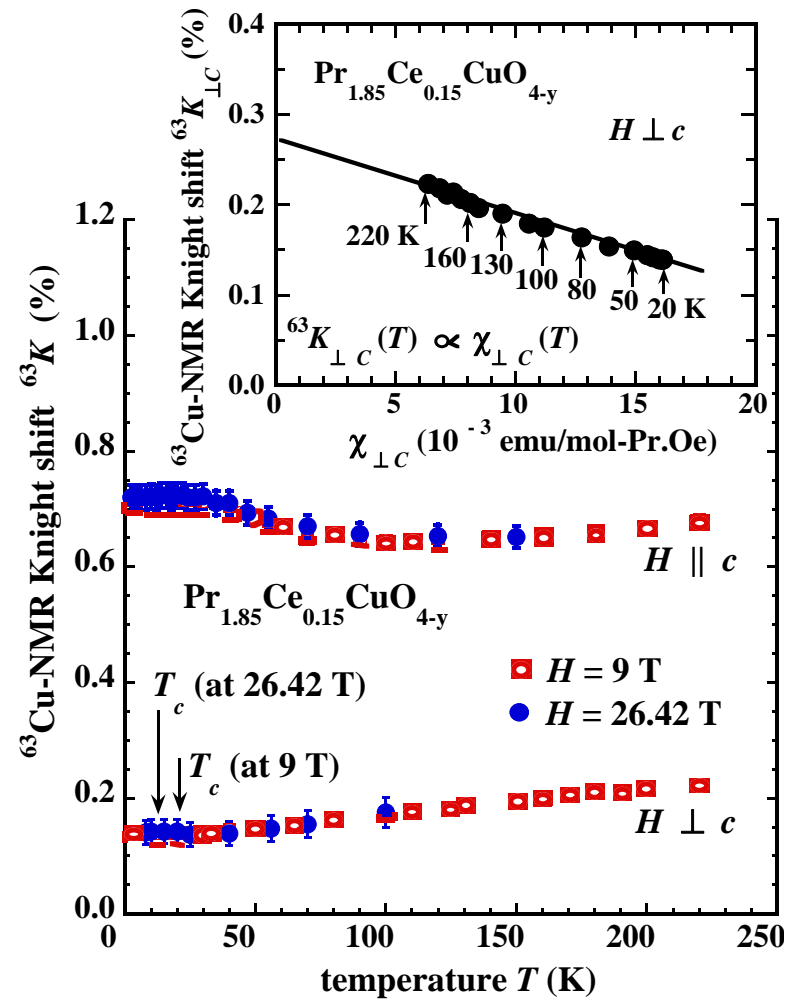

FIG. 5: (Color online) $T$-dependence of the ${ }^{63} \mathrm{Cu}-\mathrm{NMR}$ Knight shift ${ }^{63} K(T)$ of $\operatorname{Pr}_{1.85} \mathrm{Ce}_{0.15} \mathrm{CuO}_{4-y}$ (PCCO) at $H$ $=9 \mathrm{~T}$ and $H=26.42 \mathrm{~T}$. The downward arrows indicate the temperature $T_{c}$ at the corresponding field for $H \perp c$. The inset shows the linear relation of ${ }^{63} K(T)$ vs $\chi_{\perp c}(T)$ at $H \perp$ $c$, where $\chi_{\perp c}(T)$ is the $\operatorname{Pr}^{3+}$ electron paramagnetic susceptibility at $H \perp c$.

Knight shift for ${ }^{65} \mathrm{Cu}\left[{ }^{65} K(T)\right]$ (not shown) is the same as ${ }^{63} K(T)$ (for ${ }^{63} \mathrm{Cu}$ ).

For the Knight shift ${ }^{63} K(T)$, the internal static magnetic field ( $\left.H_{\text {local }}\right)$ magnitude at the ${ }^{63} \mathrm{Cu}$ can be written as, $H_{\text {local }}=H\left[1+{ }^{63} K(T)+\frac{\Delta \nu_{Q}(\Theta, T)}{\nu_{0}}\right]$, where $\frac{\Delta \nu_{Q}(\Theta, T)}{\nu_{0}}$ $=\frac{\Delta \nu_{c Q}^{(1)}(\Theta, T)+\Delta \nu_{c Q}^{(2)}(\Theta, T)}{\nu_{0}}=\frac{3}{16} \frac{\nu_{Q}^{2}}{\nu_{0}^{2}}\left(1-\cos ^{2} \Theta\right)\left(1-9 \cos ^{2} \Theta\right)$. Here $\frac{\Delta \nu_{Q}(\Theta, T)}{\nu_{0}}$ is negligible $(<0.01 \%)$ due to the very small value of $\nu_{Q}$, and ${ }^{63} K(T)$ can be expressed as $\underline{48,49}$

$$
\begin{aligned}
{ }^{63} K(T) \approx & A_{C u}^{h f} \chi_{0}+\left(A_{P r}^{h f}+A_{P r}^{D i p}\right) \chi(T) \\
& +4 \pi\left(\frac{1}{3}-D\right) \frac{\chi_{\text {sample }}(T)}{N_{A} v_{P r}}+{ }^{63} K_{\text {orb }}
\end{aligned}
$$

where $A_{C u}^{h f}$ is the anisotropic hyperfine coupling to the $\mathrm{Cu}^{2+}$ conduction electron spins in the $\mathrm{CuO}_{2}$-plane. $A_{P r}^{\text {hf }}$ and $A_{P r}^{D i p}$ are the contact hyperfine and dipolar couplings to the $\mathrm{Pr}^{3+}$ electron paramagnetic moment, respectively. Here we use $\chi_{0} \approx 4 \times 10^{-5}(\mathrm{emu} / \mathrm{mol}-\mathrm{Cu}$.Oe) $\stackrel{48,50}{,}$ which is the static Pauli spin susceptibility of the conduction electrons from the $\mathrm{CuO}_{2}$ planes (note, the value of $\chi_{0}$ is very small, and thus we expect the effect to it from the lack of apical oxygen in the PCCO crystal lattice is 
not significant), $\chi(T)$ is the $\operatorname{Pr}^{3+}$ electron paramagnetic susceptibility, and $\chi_{\text {sample }}(T)$ is the sample magnetic susceptibility $\left[\chi_{\text {sample }}(T) \approx \chi(T)+\chi_{0}\right]$. The 3rd term in Eq. (3) is the correction due to bulk demagnetization and Lorentz fields ${ }^{30}, v_{P r}$ is the unit cell volume $/ \operatorname{Pr}^{3+}, D$ $\approx 0.04$ and 0.93 at $H \perp c$ and $H \| c$, respectively, due to the sample size from our estimate,, 51 and the last term ${ }^{63} K_{\text {orb }}$ comes from the $T$-independent $\mathrm{Cu}^{2+} 3 d$-orbital contribution.

From the PCCO lattice structure we calculated 52 that $A_{P r, \| c}^{D i p}=+6.2 \mathrm{kG} / \mu_{B}($ or $0.124 \mathrm{~T})$, and $A_{P r, \perp c}^{D i p}=-$ $3.1 \mathrm{kG} / \mu_{B}$ (or $-0.062 \mathrm{~T}$ ) (note, $\left.1 \mathrm{~T}=5 \mathrm{kG} / \mu_{B}\right)$. We also estimated that $A_{C u, \| c}^{h f}=-100 \mathrm{kG} / \mu_{B}$ (or -20 $\mathrm{T}$ ), and $A_{C u, \perp c}^{h f}=+180 \mathrm{kG} / \mu_{B}($ or $+36 \mathrm{~T})$, with the consideration of the measured normal state ${ }^{63} \mathrm{Cu}-\mathrm{NMR}$ spin-lattice relaxation. ${ }^{51,52}$

Thus, with the fits to Eq. (3) and the analysis as that shown by the solid line in the inset of Fig. 4 for the Knight shift at $H \perp c$, we obtained ${ }^{63} K_{o r b, \perp c}=(0.18 \pm$ $0.01) \%$, and $A_{P r, \perp c}^{h f}=(-4.25 \pm 0.1) \mathrm{kG} / \mu_{B}[$ or $-(0.85$ $\pm 0.02)(\mathrm{T})]$. Similarly, for $H \| c$, we have ${ }^{63} K_{\text {orb }, \| c}=$ $(0.84 \pm 0.01) \%$, and $A_{P r, \| c}^{h f}=(-4.20 \pm 0.2) \mathrm{kG} / \mu_{B}[$ or $-(0.85 \pm 0.04)(\mathrm{T})]$ (here the subscripts / superscripts of $\| c$ and $\perp c$ denote the $H$ direction relative to the lattice $c$-axis). This give a high anisotropy ratio of ${ }^{63} K_{o r b, \| c} /$ ${ }^{63} K_{\text {orb }, \perp c}=4.6 \pm 0.1$.

Therefore, this indicates that the ${ }^{63} \mathrm{Cu}-\mathrm{NMR}$ Knight shift is dominated by the $T$-independent anisotropic orbital shifts, ${ }^{63} K_{\text {orb }, \| c}($ at $H \| c)$ and ${ }^{63} K_{\text {orb }, \perp c}($ at $H \perp c)$, arising from the hyperfine to the $\mathrm{Cu}^{2+} 3 d$-orbitals, while the weak $T$-dependence of the Knight shift is determined by the isotropic contact hyperfine coupling to the $\operatorname{Pr}^{3+}$ paramagnetic spins $\left(A_{P r, \| c}^{h f}=A_{P r, \perp c}^{h f} \approx-4.2 \mathrm{kG} / \mu_{B}\right.$ from above), to which the dipolar coupling $\left(A_{P r, \| c}^{D i p}\right.$ and $\left.A_{P r, \perp c}^{D i p}\right)$ is only $\sim 14.5 \%$ and $7.2 \%$ at $H \| c$ and $H \perp c$, respectively. The negative value of $A_{P r, \| c}^{h f}\left(A_{P r, \perp c}^{h f}\right)<0$, indicates an antiferromagnetic character for the coupling.

\section{C. ${ }^{63} \mathrm{Cu}-\mathrm{NMR}$ linewidth}

Figure 6 shows the $T$-dependence of the ${ }^{63} \mathrm{Cu}-\mathrm{NMR}$ central linewidth (FWHM), $\Delta f(T)$, plotted as $\Delta f(T) / H$ in units $\mathrm{kHz} / \mathrm{T}$ vs $T$. As described above, the central line has a magnetic origin, and it is inhomogeneously broadened upon cooling in temperature. Thus considering the ${ }^{63} \mathrm{Cu}$-NMR Knight shift, in which the anisotropic hyperfine from the dipolar field of the $\mathrm{Pr}^{3+}$ paramagnetic spins and from the $\mathrm{Cu}^{2+}$ conduction electron spins (Pauli) are not significant, we expect the central linewidth to be written as

$$
\Delta f(T) / H \approx A_{\text {iso }}^{P r} \chi(T)+C_{\text {orb }}+\delta f_{\text {disorder }}(T),
$$

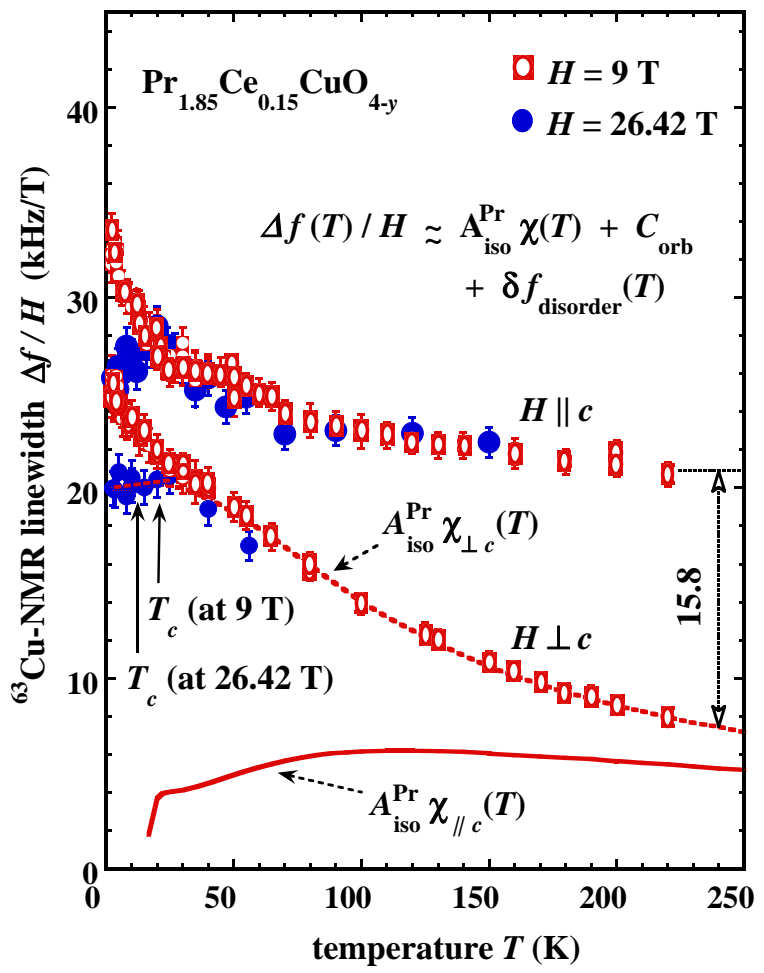

FIG. 6: (Color online) T-dependence of the ${ }^{63} \mathrm{Cu}-\mathrm{NMR}$ central linewidth (FWHM) divided by $H, \Delta f(T) / H$, of $\operatorname{Pr}_{1.85} \mathrm{Ce}_{0.15} \mathrm{CuO}_{4-y}(\mathrm{PCCO})$ at $H=9 \mathrm{~T}$ and $H=26.42$ T. The dashed (at $H \perp c$ ) and solid (at $H \| c$ ) lines indicate the contribution from the anisotropic $\operatorname{Pr}^{3+}$ magnetic susceptibility.

where $A_{\text {iso }}^{P r}$ is an isotropic constant, $C_{\text {orb }}$ is the $T$ independent anisotropic $\mathrm{Cu}^{2+} 3 d$-orbital contribution, and $\delta f_{\text {disorder }}(T)$ is due to magnetic disorder (if any).

As shown by the solid and dashed lines in Fig. 4, the linewidth data can be well-fitted with Eq. (4) as

$$
\begin{array}{rlr}
\Delta f_{\| c}(T) / H & \approx A_{\text {iso }}^{P r} \chi_{\| c}(T)+C_{\text {orb }, \| c},(T \geq 100 \mathrm{~K})(5) \\
\Delta f_{\perp c}(T) / H & \approx A_{\text {iso }}^{P r} \chi_{\perp c}(T), \quad(T \geq 20 \mathrm{~K})(6)
\end{array}
$$

where the fitted values of $A_{\text {iso }}^{P r} \approx 1.17 \times 10^{3}$ $[(\mathrm{kHz} / \mathrm{T}) .(\mathrm{mol} . \mathrm{Pr} . \mathrm{Oe} / \mathrm{emu})], \quad C_{\text {orb }, \| c} \sim 15.8 \mathrm{kHz} / \mathrm{T}$, $C_{\text {orb }, \perp \mathrm{c}} \sim 0, \delta f_{\text {disorder }}^{\| c}(T) \sim 0$, and $\delta f_{\text {disorder }}^{\perp c c}(T) \sim 0$, in the temperature range specified above in Eqs. (5)-(6).

Thus Fig. 6 [ Eqs. (4) - (5) ] reveals that, 1) the ${ }^{63} \mathrm{Cu}-$ NMR central linewidth $\Delta f(T)$ is essentially proportional to the applied magnetic field $H$ (because of its magnetic origin) except for the development of possible magnetic disorder at low- $T(<\sim 25 \mathrm{~K}), 2)$ at $H \| c$ the linewidth is dominated by the anisotropic $\mathrm{Cu}^{2+} 3 d$-orbital contributions (due to the $\mathrm{Cu}^{2+}$ orbital moments), and 3) at $H$ $\perp c$ the linewidth is almost completely determined by the isotropic contact hyperfine coupling to the $\mathrm{Pr}^{3+}$ paramagnetic moments, i.e., there is a negligible contribution from the $\mathrm{Cu}^{2+} 3 d$-orbital to the internal static magnetic field distribution at the $\mathrm{Cu}$ at $H \perp c$. But the $\mathrm{Cu}^{2+}$ $3 \mathrm{~d}$-orbital contribution always dominates the ${ }^{63} \mathrm{Cu}-\mathrm{NMR}$ 
Knight shift (internal static magnetic field magnitude) at both $H \perp c$ and $H \| c$. This local magnetic field environment at the $\mathrm{Cu}$ in PCCO is very different from that in the hole-doped cuprate HTSCs, $\frac{48}{}$ where effects from ions of large spin paramagnetic moment (like $\mathrm{Pr}^{3+}$ in $\mathrm{PCCO}$ or $\mathrm{Nd}^{3+}$ in $\mathrm{NCCO}$ ) do not exist.

The origin for the development of possible magnetic disorder at low $T$ (seen from the linewidth) is not clear, even though it could come from minor impurity oxygen 53 trapped in the sample during the sample synthesis process.

\section{D. ${ }^{63} \mathrm{Cu}$ 3d-orbital energy splitting}

Finally, the parameters for the energy splitting of the $\mathrm{Cu}^{2+} 3 d$ orbitals in the $\mathrm{CuO}_{2}$-plane can be obtained ${ }^{33,34}$ through the orbital Knight shift anisotropy as 33,54

$$
\begin{aligned}
{ }^{63} K_{\mathrm{orb}, \| \mathrm{c}} /{ }^{63} K_{\mathrm{orb}, \perp \mathrm{c}} & =4\left(E_{x z}-E_{x^{2}-y^{2}}\right) /\left(E_{x y}-E_{x^{2}-y^{2}}\right), \\
E_{x z} & =E_{y z},
\end{aligned}
$$

where $E_{x y}, E_{x z}, E_{y z}$, and $E_{x^{2}-y^{2}}$ are the energy levels of the $\mathrm{Cu}^{2+} \mathrm{d}_{x y}, \mathrm{~d}_{x z}, \mathrm{~d}_{y z}$ and $\mathrm{d}_{x^{2}-y^{2}}$ orbitals, respectively.

By using the obtained anisotropy ratio of ${ }^{63} K_{\text {orb }, \| c} /{ }^{63} K_{\text {or } b, \perp c}=4.6 \pm 0.1$, we have the energy state of the $\mathrm{Cu}^{2+} 3 d$-electrons as $\frac{E_{x z}-E_{x^{2}-y^{2}}}{E_{x y}-E_{x^{2}-y^{2}}}=1.15 \pm 0.01$, i.e., $E_{x z}=E_{y z}>E_{x y}$. This agrees well with the theoretical calculation 54 and observation 55 regarding the $\mathrm{Cu}^{2+} 3 d$-orbital energy levels of $E_{x z}, E_{y z}$ and $E_{x y}$ relative to the ground level $E_{x^{2}-y^{2}}$, suggesting a similar high anisotropy of the $\mathrm{Cu}^{2+} 3 d$ orbital shift and similar electronic energy state of the $\mathrm{Cu}^{2+}$ electron itself in the $\mathrm{CuO}_{2}$-plane to those in the hole-doped cuprate HTSCs. 55

\section{CONCLUSION}

In summary, a very small NQR frequency $\nu_{Q} \sim 2.2$ $\mathrm{MHz}$ is obtained with the observation of an unusual
${ }^{63,65} \mathrm{Cu}-\mathrm{NMR}$ spectrum, which shows a very small electric field gradient (EFG) and an extremely wide continuous distribution of it $\left(\Delta \nu_{Q} \sim 18 \mathrm{MHz}\right)$ at the copper in PCCO. Upon cooling in temperature, the distribution of EFG becomes significantly narrower below $20 \mathrm{~K}$ at $H$ $c$ where the superconductivity is completely suppressed, indicating a significant change in the charge distribution modulation at the $\mathrm{Cu}$ site. Other experimental techniques are needed to verify whether this is due to $\mathrm{CO}$ or a different type of charge distribution modulation.

The ${ }^{63,65} \mathrm{Cu}-\mathrm{NMR}$ Knight shift and the central linewidth are proportional to the externally applied magnetic field, with an orbital shift anisotropy of $\sim 4.6$. We find that the magnitude of the internal static magnetic field at the copper at both $H \perp c$ and $H \| c$ is dominated by the $T$-independent anisotropic hyperfine coupling to the $\mathrm{Cu}^{2+} 3 d$ orbitals, while its weak $T$-dependence is mainly determined by the isotropic contact hyperfine coupling to the paramagnetic $\mathrm{Pr}^{3+}$ electron spins, which is also responsible for the full distribution of the internal static magnetic field at the copper at $H \perp c$. But at $H \|$ $c$, the distribution of the internal static magnetic field at the copper is dominated by the $\mathrm{Cu}^{2+} 3 d$-orbital contributions through anisotropic hyperfine couplings. Thus, unlike the $\mathrm{Cu}^{2+} 3 \mathrm{~d}$ orbitals, the $\mathrm{Cu}^{2+}$ spins provide a small contribution to the internal static magnetic field at the copper in PCCO. This unusual internal static electric and magnetic field environment at the copper in cuprate HTSCs may provide new insight into the understanding of the high- $T_{c}$ superconductivity.

\section{Acknowledgments}

The work at NHMFL was supported by NSF under Cooperative Agreement No. DMR-0654118 and the State of Florida, at UM by DMR-1104256 (RLG), at University of West Florida by SCA/2009-2012 (G. Wu) and at UCLA by NSF Grants DMR-0334869 (WGC). We thank Stuart E. Brown for helpful discussions and support.
1 E. H. da Silva Neto, R. Comin, F. He, R. Sutarto, Y. Jiang, R. L. Greene, G. A. Sawatzky, and A. Damascelli, Charge ordering in the electron - doped $\mathrm{Nd}_{2-x} \mathrm{Ce}_{x} \mathrm{CuO}_{4}$, submitted to Science, 2014.

2 M. P. M. Dean et al., Nat. Materials, 12, 1019 (2013).

3 D. J. Scalapino, Rev. Mod. Phys. 84, 1383 (2012).

4 D. Pines, Physica B 163, 78 (1990); in High Temperature Superconductivity, edited by K. S. Bedell et al. (Addison-Wesley, New York, 1990), p. 392.

5 P. W. Anderson, Theory of Superconductivity in the High $-T_{c}$ Cuprates (Princeton University Press, Princeton, NJ, 1997).

6 A. G. Loeser, Z.-X. Shen, D. S. Dessau, D. S. Marshall, C. H. Park, P. Fournier, and A. Kapitulnik, Science 273, 325
(1996).

7 H. Ding et al., Nature (London) 382, 51 (1996).

8 A. Mann, Nature 475, 280 (2011).

9 T. P. Croft, C. Lester, M. S. Senn, A. Bombardi, and S. M. Hayden, Phys. Rev. B 89, 224513 (2014).

10 D. H. Torchinsky, F. Mahmood, A. T. Bollinger, I. Božvić, and N. Gedik, Nat. Materials 12, 387 (2013).

11 J. Chang et al., Phys. Rev. B 78, 104525 (2008).

12 G. Ghiringhelli et al., Science 337, 821 (2012).

13 T. Wu et al., Nat. Commun. 4, 3113 (2013); Nature 477, 191 (2011).

14 D. Leboeuf, S. Krämer, W. N. Hardy, R. Liang, D. A. Bonn, and C. Proust, Nat. Phys. 9, 79 (2013).

15 J. Chang et al., Nat. Phys. 8,871 (2012). 
16 C. V. Parker et al., Nature 488, 677 (2010).

17 J. E. Hoffman, E. W. Hudson, K. M. Lang, V. Madhavan, H. Eisaki, S. Uchida, and J. C. Davis, Science 295, 466 (2002).

18 A. J. Achkar et al., Phys. Rev. Lett. 109, 167001(2012).

19 B. Lake et al., Nature 415, 299 (2002).

${ }^{20}$ J. P. Hinton, J. D. Koralek, G. Yu, E. M. Motoyama, Y. M. Lu, A. Vishwanath, M. Greven, and J. Orenstein, Phys. Rev. Lett. 110, 217002 (2013).

21 M. Yi et al., Nat. Commun. 5, 3711 (2014).

22 B. Lorenz, A. M. Guloy, Paul C. W. Chu, Int. J. Mod. Phys. B 28, 1430011 (2014).

23 P. Doan et al., J. Am. Chem. Soc 134, 16520 (2012).

24 Y. Tokura et al., Nature 337, 345 (1989); H. Takagi, S. Uchida, and Y. Tokura, Phys. Rev. Lett. 62, 1197 (1989).

${ }^{25}$ K. Jin, N. P. Butch, K. Kirshenbaum, J. Paglione, and R. L. Greene, Nature 476, 73 (2011).

26 N. P. Armitage, P. Fournier, and R. L. Greene, Rev. Mod. Phys. 82, 2421 (2010).

27 Our upper critical field $H_{c 2}$ vs $T$ measurement shows $H_{c 2, \perp c}(0) / H_{c 2, \| c}(0)=42 \mathrm{~T} / 6 \mathrm{~T}$, where $H_{c 2, \perp c}(0)$ and $H_{c 2, \| c}(0)$ are $H_{c 2}$ values at $H \perp c$ and $H \| c$ when $T$ $\rightarrow 0$, respectively. At $\perp c, T_{c}=20 \mathrm{~K}$ and $12 \mathrm{~K}$ for $H=9$ $\mathrm{T}$ and $26.42 \mathrm{~T} \perp c$, respectively.

28 C. Weber, K. Haule, and G. Kotliar, Nat. Phys. 6, 574 (2010).

29 C. R. Rotundu et al, Phys. Rev. B 87, 024506 (2013).

30 C. P. Slichter, Principles of Magnetic Resonance, 3rd ed. (Springer, Berlin, 1989).

31 K. Kumagai, and Y. Nakamura, Physica C 157, 307 (1989).

${ }^{32}$ W. W. Warren, Jr., R. E. Walstedt, G. F. Brennert, R. J. Cava, R. Tycko, R. F. Bell, and G. Dabbagh, Phys. Rev. Lett. 62, 1193 (1989).

33 S. E. Barrett, D. J. Durand, C. H. Pennington, C. P. Slichter, T. A. Friedmann, J. P. Rice, and D. M. Ginsberg, Phys. Rev. B 41, 6283 (1990).

34 C. H. Pennington, D. J. Durand, C. P. Slichter, J. P. Rice, E. D. Bukowski, and D. M. Ginsberg, Phys. Rev. B 39, 2902 (1989).
35 T. Wu et al., Nat. Commun. 4, DOI: 10.1038/ncomms3113 (2013).

36 T. Wu et al., Nature 477, 191 (2011).

37 G. V. M Williams, S. Krämer, R. Dupree, and A. Howes, Phys. Rev. B 69, 134504 (2004).

38 A. Kobayashi, A. Tsuruta, T. Matsuura, and Y. Kuroda, J. Phys. Soc. Jpn. 71, 1640 (2002).

39 M. Jurkutat et al., https://arxiv.org/abs/1403.6289 (Mar. $25,2014)$.

40 M. Jurkutat, J. Haase, and A. Erb, J. Supercond. Nov. Magn. 26, 2685 (2013).

41 J. L. Peng, Z.Y. Li, and R. L. Greene, Physica C 177, 79 (1991).

42 M. Brinkmann et al., T. Rex, H. Bach, and K. Westerholt, J. Cryst. Growth 163, 369 (1996).

43 W. G. Clark, M.E. Hanson, F. Lefloch, and P. Ségransan, Rev. Sci. Instrum. 66, 2453 (1995).

44 G. Gaidos, Ph.D. dissertation, University of California, Los Angeles (UCLA), 2008.

45 M. Abe, K. Kumagai, S. Awaji, and T. Fujita, Physica C 160, 8 (1989).

46 Y. Kohori,T. Sugata, H. Takenaka, T. Kohara, Y. Yamada, J. T. Markert, and M. B. Maple, J. Phys. Soc. Jpn. 58, 3493 (1989).

47 A. Yu. Zavidonov et al., SFKT 3, 1597 (1990).

48 M. Mehring, Appl. Magn. Reson. 3, 383 (1992).

49 J. E. Sonier, K. F. Poon, G. M. Luke, P. Kyriakou, R. I. Miller, R. Liang, C. R. Wiebe, P. Fournier, and R. L. Greene, Phys. Rev. Lett. 91, 147002 (2003).

50 L. F. Mattheiss, Phys. Rev. Lett. 58, 1028 (1987).

51 Guoqing Wu et al., unpublished.

52 S. E. Brown, Notes on Knight shift analysis (unpublished).

${ }^{53}$ K. Miyagawa, H. Kawamura, K. Kanoda, Y. Onose, Y. Taguchi, and Y. Tokura, Physica C 388-389, 253 (2003).

54 A. K. McMahan, R. M. Martin, and S. Satpathy, Phys. Rev. B 38, 6650 (1988).

55 J. A. Leiro, F. Werfel, and G. Dräger, Phys. Rev. B 44, 7718 (1991). 